\title{
Large eddy simulation of particle laden jet flow with aerodynamic three-way coupling
}

\author{
A. Jadoon \& J. Revstedt \\ Lund University, Department of Energy Sciences, Sweden
}

\begin{abstract}
A model to incorporate for aerodynamic 3-way (indirect) coupling between mono dispersed particles is proposed. The model is applied on particle laden jet flow and the results are compared with 2 -way coupling. The particle drag coefficients are corrected based on the relative position of the particles. The correction factor is obtained from the pre-computed lookup tables. The particles are tracked by Lagrangian particle tracking (LPT), whereas the continuous phase is modelled by Large eddy simulation (LES). Particle mean axial velocity is found to be large in 3 -way coupling which results in more particle penetration in the streamwise direction due to less momentum transfer of the particles to the fluid phase. The fluid velocity is also observed to be higher in case of 3-way coupling due to high mass loading which results in reduced axial fluid velocity fluctuations. The void faction is also found to be higher in 3-way coupling due to particle entrainment and less interaction with turbulence.
\end{abstract}

Keywords: LES/LPT, turbulent jet, three-way coupling, drag correction.

\section{Introduction}

Particle laden jet flows remained an interesting and important topic due to its industrial and engineering applications like pulverized-coal combustors, cyclone separators, combustion etc. An important issue often neglected in the moderately dense flows is the aerodynamic (indirect) interaction between the particles i.e. three-way coupling. The addition of particles to turbulent flows can change the flow characteristics like turbulent intensities even at very low volume fraction. Therefore taking the particle interaction into account in such flows still needs to be addressed.

Previously most of the research both experimentally and numerically has been carried out considering one-way and two-way coupling. Longmire and Eaton [1] 
studied experimentally the dispersion of particles when they interact with turbulent jet dominated with vortex ring structures. They further confirmed that the local dispersion and concentration of particles are governed by convection due to large scale structures. Kulick et al. [2] experimentally investigated the gas phase turbulent flows with heavy particles of mass loading up to $80 \%$. The fluid turbulence is found to be attenuated when particles are added depending on particle relaxation time to turbulent scale and solid mass loading. Boivin et al. [3] investigated the feasibility of using Large eddy simulation (LES) when the gas flow turbulence is modified due to momentum exchange of particles. They conducted a priori tests of sub-grid scale models by using the results obtained from DNS. He found a good agreement with DNS data by using dynamic sub-grid models. Jin et al. [4] using LES predict the particle dispersion patterns when particles with different Stokes number (based on particle diameter) are injected. Their results are in good agreement with the experimental work.

Regarding the inter-particle interaction, Tanaka and Tsuji [5] simulated the two phase (gas-solid) flow considering inter-particle collisions. They used the deterministic method to calculate the inter-particle collision where inter-particle collision is described by hard sphere model. They reported that even in dilute conditions (volume fraction $\left(10^{-4}\right)$ ), the inter-particle collision has large effect on particle diffusion. Sommerfeld and Zivkovik [6] applied the stochastic collision model in a dilute phase pneumatic conveying through pipe systems. They also showed that the effect of inter-particle collision is significant even at low overall mass loading for the development of particle concentration profiles. Oesterle and Petitjean [7] simulated the gas-solid flow to demonstrate the significance of inter-particle collision in horizontal pipes. The other contribution concluding the importance of inter-particle collision are Lun and Lui [8], Sakiz and Simonin [9], Sommerfeld [10]. Sommerfeld [11] also applied the stochastic model of particle collision to isotropic homogeneous turbulence flow and obtained reliable results. More recently Yan et al. [12], conducted the Direct numerical simulation (DNS) of three dimensional two phase jet. The inter-particle collision was described by deterministic hard-sphere model. The study was only focused on inter-particle collision by neglecting the two-way coupling. They found that occurrence of interparticle collision correlates well with local particle concentration, though average concentration of the particles is lower in the whole field. They also reported that the relation between the average inter-particle collision number and stokes number is not a linear but has a local maximum.

In the present studies, only the effect of three-way aerodynamic coupling (indirect collision) in gas-solid flow is accounted by considering the flows in which number density of the particles is not high enough to have an equilibrium in interaction among particles, secondly the inter-particle distance is not large enough to neglect the particle interaction. However the direct inter-particle collisions are neglected in this study. It has been shown for particle Reynolds number $<200$, that drag of particle placed in the wake of reference particle is affected even up-to $6 d_{p}\left(d_{p}\right.$ is the diameter of the particle) distance, Prahl et al. [13]. Prahl et al [14], Jadoon et al. [15] also found that even for high particle Reynolds number 
i.e. up-to 600 , the drag of trailing particle in the wake of reference particle is still 90 percent of the drag of the single particle at $9 d_{p}$. Hence the particle interaction is important in dilute flows also. A model is proposed to account the three-way coupling. Salewski and Fuchs [16] applied this drag correction technique to a spray in cross-flow. They corrected the drag of the particles up to particle Reynolds number of 200 . They found $40 \%$ decrease in the average drag of particles in the near-field (dense) jet due to aerodynamic interaction. However it decreases to $10 \%$ at $15 D_{N}$ ( $D_{N}$ is the diameter of the nozzle) downstream. The technique is applied to particle laden jet flow in order to analyze the effect of the model on velocities of both discrete and continuous phase, particle dispersion, velocity fluctuations etc. The current study is the step towards the complete parameterization of the model which includes the effect of particle size, stokes number, turbulent Reynolds number and mass loading. The data is available for particles Reynolds number up-to 600 , however in the current study the only particle Reynolds numbers up to 200 are achieved.

\section{Mathematical models}

The continuous phase is modelled by LES whereas the particles are tracked by Lagrangian particle tracking (LPT)

\subsection{Modelling of continuous phase}

In LES, the large scales are resolved and small scales are modelled. As large scales are assumed to be most important ones because of energy and momentum transport in turbulent flows. The space filtered non-dimensional Navier-Stokes equation for incompressible flows can be written as:

$$
\begin{gathered}
\frac{\partial \bar{u}_{i}}{\partial x_{i}}=0 \\
\frac{\partial \overline{u_{i}}}{\partial t}+\overline{u_{j}} \frac{\partial \bar{u}_{i}}{\partial x_{j}}=-\frac{\partial \bar{p}}{\partial x_{i}}+\frac{1}{R e}\left(\frac{\partial^{2} \overline{u_{i}}}{\partial x_{j}{ }^{2}}\right)-\frac{\partial \tau_{i j}}{\partial x_{j}}
\end{gathered}
$$

Equations (1) and (2) govern the resolved scale motion. The effect of the small scales are modelled through sub-grid scale (SGS) stresses.

$$
\tau_{i j}=\overline{u_{i} u_{j}}-\overline{u_{i}} \overline{u_{j}}
$$

In the current study, no explicit SGS model is applied instead the properties of the numerical scheme are used to achieve the necessary dissipation of energy at small scales, Rai and Moin [17]. 


\subsection{Particle equation of motion}

Lagrangian particle tracking based on momentum equation for particle is stated as:

$$
m \frac{d \vec{u}_{p}}{d t}=\vec{F}
$$

In the above equation, $F$ is the forces acting on the particle and $m$ is its mass. As the ratio of particle to air density is high i.e. approx 2000, therefore only the drag and the gravity forces are considered. The equation for the drag force in nondimensional form:

$$
F_{D}=-\frac{3}{4} \frac{\rho_{c}}{\rho_{p}} \frac{1}{d_{p}^{*}} C_{D}\left|U_{r}^{*}\right| U_{r}^{*}
$$

where $\rho_{c}$ and $\rho_{p}$ are the densities of continuous (fluid) and dispersed (particle) phase respectively. $d_{p}^{*}$ is the non-dimensional diameter of the particle scaled with the diameter of the nozzle $D_{N}$ and $U_{r}^{*}$ is non-dimensional relative velocity scaled with inlet air velocity $U_{o} . C_{D}$ is the drag coefficient and the correlation is given by Schiller and Nauman [18] as:

$$
\begin{gathered}
C_{D}=\frac{24}{R e_{p}}, \text { for } R e_{p}<<1 \\
C_{D}=\frac{24}{R e_{p}}\left(1+0.15 \operatorname{Re}_{p}{ }^{0.687}\right), \text { for } R e_{p} \leq 1000 \\
C_{D}=0.44, \text { for } 1000 \leq R e_{p} \leq 100000
\end{gathered}
$$

Here $R e_{p}$ is the particles Reynolds number and defined as:

$$
R e_{p}=\frac{d_{p}\left|V_{i}-u_{i}\right|}{\nu_{c}}
$$

Similarly the non-dimensional gravity term is as follows:

$$
F_{\text {grav }}=\left(1-\frac{\rho_{c}}{\rho_{p}}\right) \frac{1}{F r^{2}}
$$

where $F r$ is the Froud number defined as ratio between inertial to gravitational forces and expressed as $U_{o} / \sqrt{g D_{N}}$, where $g$ is the acceleration due to gravity.

\section{Numerical methods}

The numerical scheme used in this current study is based on Olsson and Fuchs [19]. The Governing equations are discretized on a staggered cartesian grid. Fourth order central finite difference scheme is used for approximation of the equation except for convective term. The convective terms are discretized using third order upwind based schemes Rai and Moin [17]. The governing equations are solved 
using a split solver; explicit and implicit. The momentum equations are integrated explicitly in time using fourth order Runge-Kutta type method. A Poison equation is solved for the pressure correction. A multi-grid scheme is used to accelerate the solution of the Poisson equation.

\section{Problem description}

In all simulations, a rectangular domain of $[8,14,8] D_{N}\left(D_{N}\right.$ is the diameter of the nozzle) corresponding to $[x, y, z]$ directions respectively is used. The jet enters at the center of the $x-z$ plane ( $x$ and $z$ being lateral coordinates) and flows in the $y$ (stream-wise) direction. The four Multi-grid levels with 96, 290 and 96 cells corresponding to $\mathrm{x}, \mathrm{y}$ and $\mathrm{z}$ directions respectively on the finer level are used. Grid stretching is used in all three coordinates for high spatial resolution ensuring that order of accuracy is maintained. At inflow, fluctuations are introduces in the radial velocities as $\pm 10 \%$ of the stream wise velocity and no slip boundary conditions at walls. At outflow both Neumann convective or non-reflective boundary conditions are tested and no difference is observed.

\subsection{Two-way coupling}

In the two-way coupling, the results are validated with the experimental work of Longmire and Eaton [1]. The jet is forced with a strouhal number $S t=0.43$. The jet Reynolds number of 19000 based on jet diameter $D_{N}$, fluid inlet velocity $U_{o}$ and fluid kinematic viscosity is used. The particle mean diameter of 0.00275 and particle to fluid density ratio of 2000 are used. The velocity of the particle is $0.55 U_{o}$. The mass loading ratio of $9 \%$ is used.

\subsection{Three-way coupling}

The aerodynamic three-way coupling is introduced by correcting the drag coefficient of the particle depending upon their relative position. The correction is done up-to particles' Reynolds number of 200. In order to achieve the particle's Reynolds number of 200 , the particle velocity is set twice the air velocity at inlet, diameter of the particle is set to 0.01 , mass loading ratio is 1.11 and Reynolds number based on air velocity and diameter of the jet is 19000 . Uniform inflow conditions are applied in 3-way coupling. The modelling of the aerodynamic threeway is achieved by using the precomputed drag correction factor $f$, and the drag coefficient in equation is then adjusted by this factor. The drag correction factors are used from the precomputed look up tables $[13,16]$.

Thus the drag force term after adjusting with correction factor $f$ becomes:

$$
F_{D}=-\frac{3}{4} \frac{\rho_{c}}{\rho_{p}} \frac{1}{d_{p}^{*}} C_{D} f\left|U_{r}^{*}\right| U_{r}^{*}
$$




\section{Results}

In the result section, all velocities are scaled with the inlet velocity $U_{o}$ and the length of the domain are scaled with the diameter of the nozzle $D_{N}$.

\subsection{Validation}

The results for two-way coupling are presented in order to validate the code. The mean properties of the discrete phase i.e. particles are compared with the experimental work of Longmire and Eaton [1]. Fig. 1(a) shows the axial mean velocity of the particles along the centerline of the jet. The velocity of the particle is under predicted by $8 \%$ in the range of shear layer $\left(\approx 4-6 D_{N}\right)$ where the potential core ends and the jet starts to spread. A high radial velocity is observed at this point i.e $Y / D_{N}=4$ which results in more spreading of particles compared to experimental data which explains the decrease in axial velocity at the end of potential core along the center line Fig. 1(b).

Fig. 1(c), (d) shows the axial velocity along the radius at different axial positions of $Y / D_{N}=2$ and $Y / D_{N}=4$ respectively. At $Y / D_{N}=2$, the results agree well with the experimental data but at $Y / D_{N}=4$ the velocity is somewhat under predicted compared to experimental data, which may be due to difference in the inlet condition of the particle velocity. Longmire and Eaton [1] do not present particle velocities at the nozzle. Therefore, instead the measured velocities at $Y / D_{N}=0.5$ are used as boundary conditions, whereas in the current simulations, the boundary conditions are set at the nozzle, which of course may be one of the reason for the discrepancies seen in Fig. 1.

The radial velocities at $Y / D_{N}=2$ and 4 are shown in Fig. 1(b). The radial velocities at $Y / D_{N}=2$ is negative for $r / D_{N}<0.35$ compared to Longmire and Eaton [1]. This may be caused by difference in entrainment between the simulation and the experiment of Longmire and Eaton [1], which in turn caused by geometrical difference. The jet is coming out from a flat surface compared to thin, sharp edged converging nozzle used in the experiments. The entrainment in the main flow is only from sides in the present case in contrast to experimental conditions where the entrainment is both from sides and from the upstream of the nozzle. This may leads to the negative radial velocity at $Y / D_{N}=2$. However, the results are in good agreement downstream at $Y / D_{N}=4$. The particles have higher radial velocities at $r / D_{N}>0.4$ showing more dispersion of the particles. Fig. 1(e), (f) shows the comparison of standard deviation of the particle axial velocity fluctuations along the radius at $Y=2 D_{N}$ and $Y=4 D_{N}$ downstream.

\subsection{Three-way coupling}

The results for the three-way coupling are presented in this section. Fig. 2(a) shows the fluid axial velocity along the centerline of the jet. The fluid velocity is found to be greater in 3-way coupling compared to 2-way coupling. However the particles experience less drag in 3-way due to drag correction and therefore less momentum 

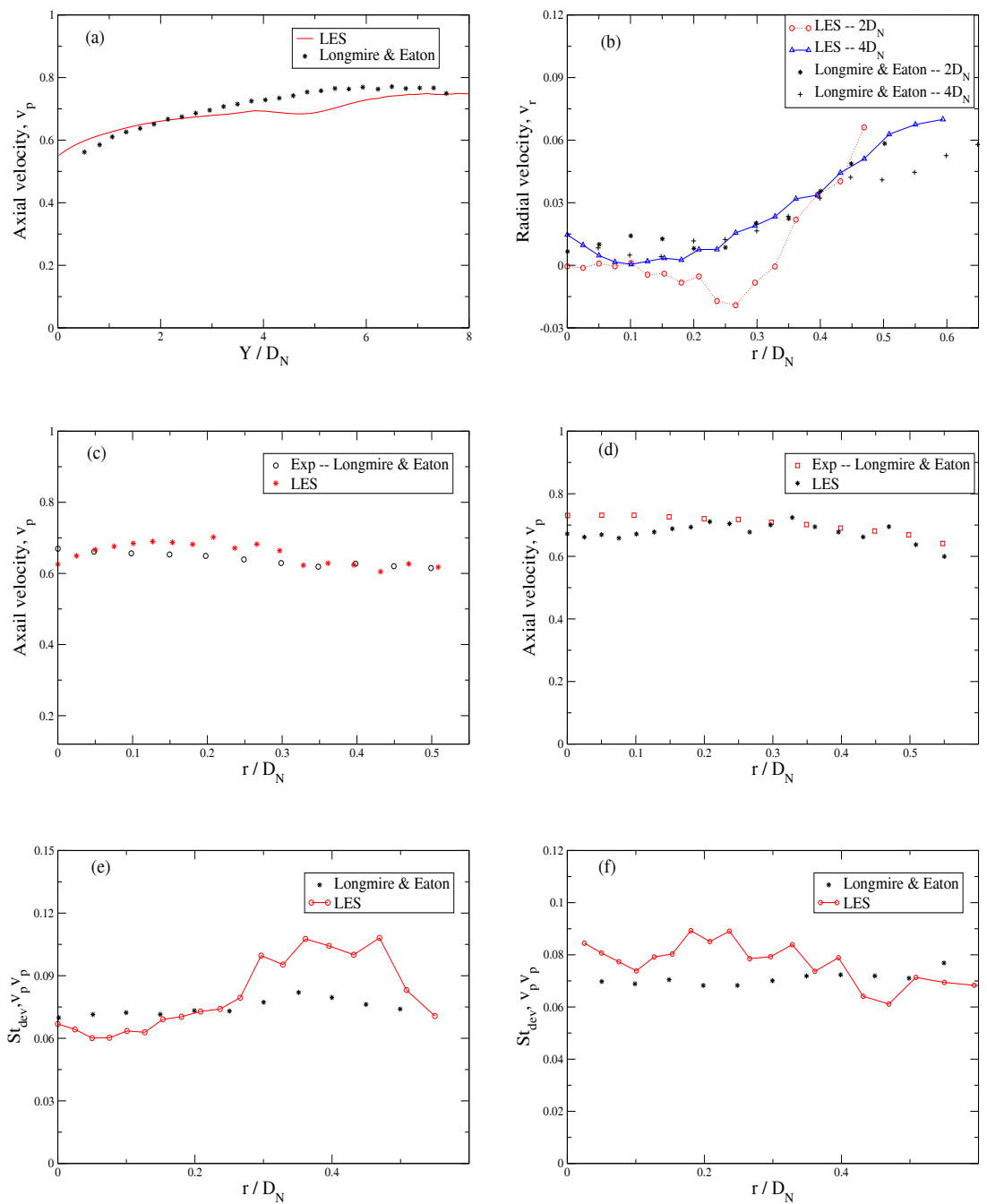

Figure 1: Particle axial velocity along the center line of the jet (a) and particle radial velocity along radius at $Y=2 D_{N}$ and $Y=4 D_{N}$ downstream (b), Particle axial velocity along the radius of the jet at axial distance of $Y=2 D_{N}$ (c) and $Y=4 D_{N}(\mathrm{~d})$, Standard deviation of the particle axial velocity fluctuations along the radius of the jet at axial distance of $Y=2 D_{N}(\mathrm{e})$ and $Y=4 D_{N}(\mathrm{f})$.

of particles is transferred to the fluid phase but on the other hand, greater volume fraction (mass loading) is found in 3-way coupling compared to 2-way (Fig. 3(a)) and it is well known conclusion that the presence of particles reduces the decay 

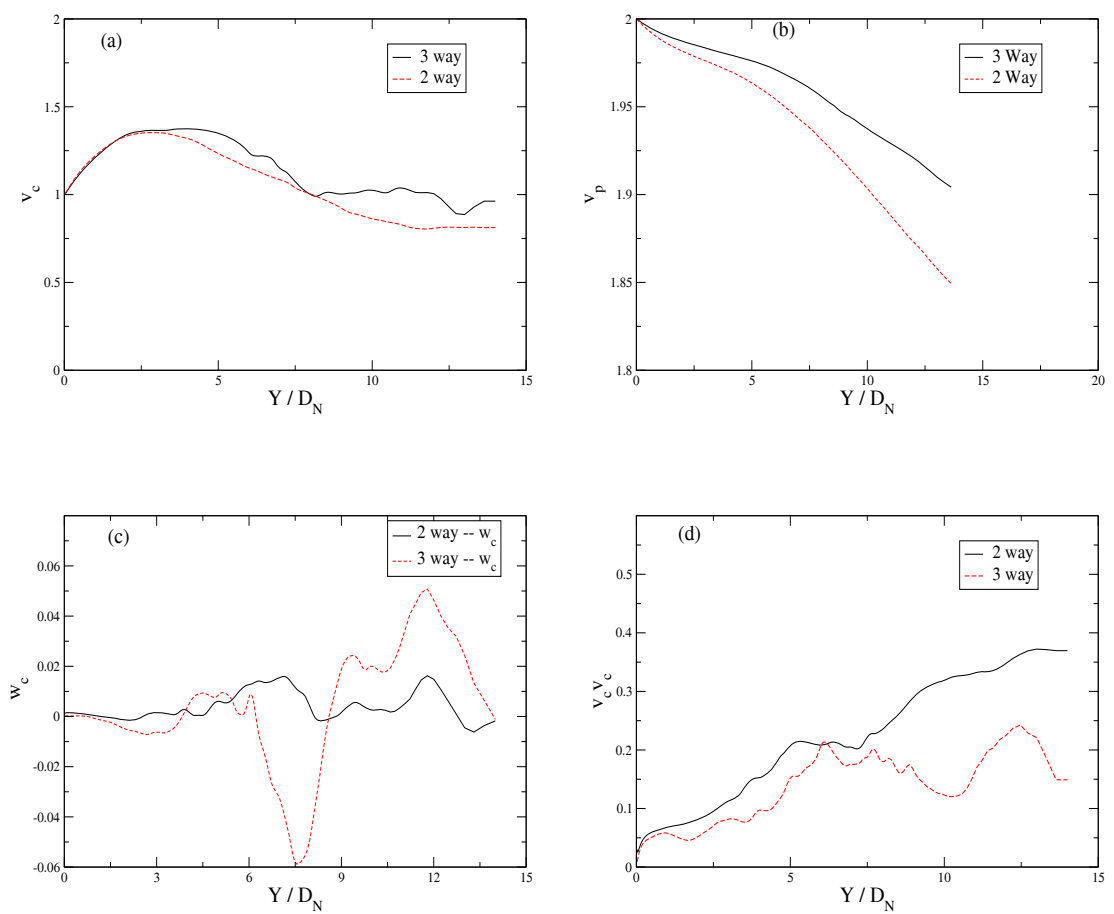

Figure 2: Fluid axial velocity (a), particle axial velocity (b), fluid radial velocity (c) and fluid axial velocity fluctuations (d) along the centerline of the jet.

of centreline air velocity. Therefore average momentum transfer in 3-way is still higher (due to high mass loading) which results in increase in gas velocity in 3-way.

The effect of less momentum transfer from particles in 3-way coupling can be seen in the mean particle velocity in Fig. 2(b), the particles penetrate more in the 3-way coupling compared to 2-way. It can be seen in Fig. 3(a) that the average number of particle (void fraction) along the center line is also greater in 3 -way coupling. This is due to the negative radial velocity attained by the fluid in 3-way coupling Fig. 2(c), therefore particles are entrained towards the center of the jet. The other reason for high mass loading may be consequence of smaller lateral diffusion due to less interaction with turbulence for 3way coupling which results in smaller source terms in the NS equations. Fig. 3(b) shows the particle Reynolds number along the centerline of the jet for 3-way coupling. The difference between the particle velocities in 2-way and 3-way coupling is greater in the region of high particle Reynolds number as the drag correction is more active in this region. The standard deviation of fluctuations of the mean axial velocity of the fluid along the center line is shown in Fig. 2(d), the fluctuations are reduced in 3-way coupling compared to 2 way as the average number of particles along the centerline in greater in 3-way coupling which helps in attenuating the turbulent 

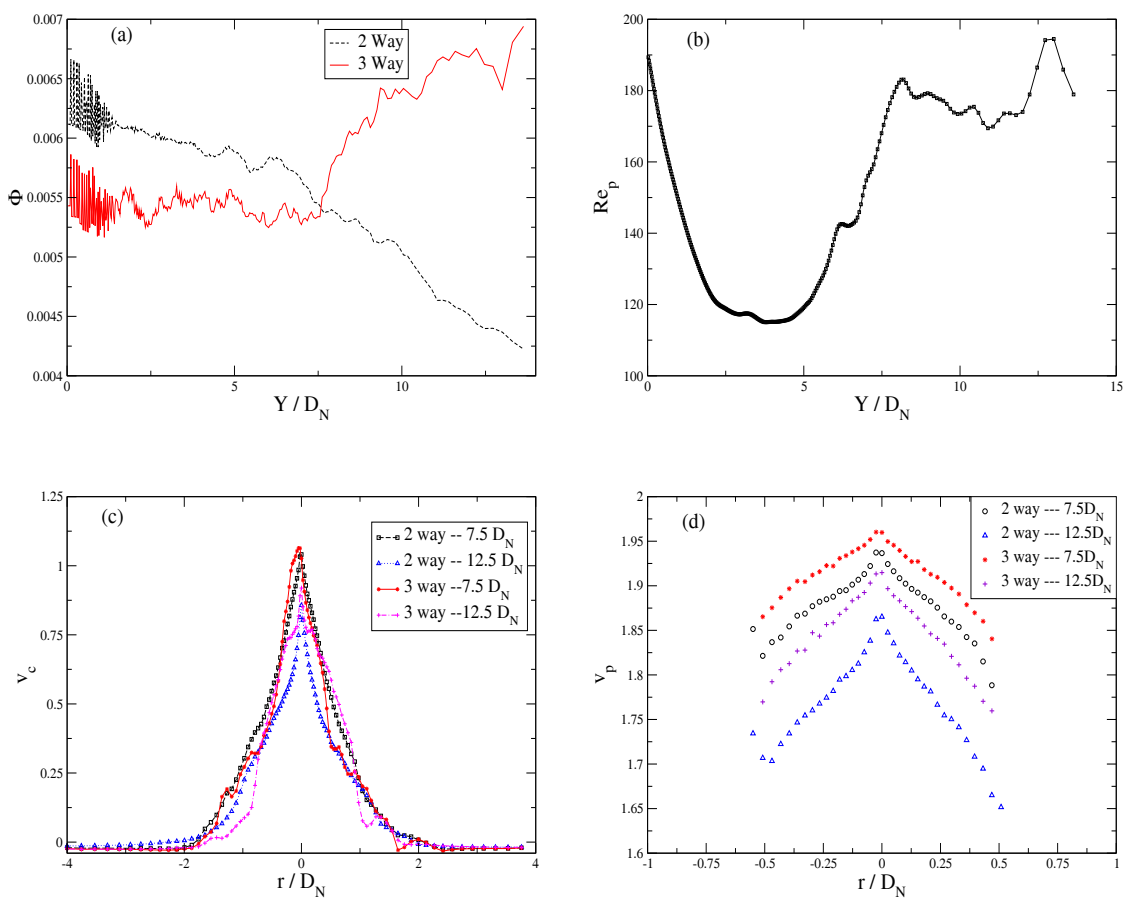

Figure 3: Void fraction (a) and particle Reynolds number (b) along the centerline of the jet, fluid axial velocity (c) and particle axial velocity (d) at $Y=$ $7.5 D_{N}$ and $12.5 D_{N}$.

intensity in 3-way case. The radial velocity component is plotted in Fig. 2(c), it can be seen that the decrease in fluid axial velocity at $7.5 D_{N}$ in Fig. 2(a) is due to the negative radial velocity gained by the fluid at this point which interferes to the axial velocity progress. However the radial velocity becomes positive afterwards and as a result axial velocity also increases again.

The mean axial velocities of both fluid and particles are shown in the Fig. 3(c) and Fig. 3(d) respectively. The difference in the particle velocities in 2-way and 3-way coupling can be easily observed. However, there is no difference along the radius of the jet is observed in both 2-way and 3-way coupling. The particles used in the simulation are heavy and bigger in size with Stokes number $>170$. The particles are merely affected by the continuous phase as they have very little time to respond to the fluid and tend to keep their initial velocity. However even with such high stokes number, the difference between the 2-way and 3-way coupling is obvious.

\section{Conclusion}

A model based on aerodynamic 3-way coupling is applied to a particle laden jet flow. The mean particle and fluid velocities are compared with the 2-way coupled 
flow. The results shows difference in mean properties of both particle and fluid properties when 3-way coupling is applied. Particles penetrate more downstream in the 3-way coupled flow as the particles experience less drag compared to 2-way coupled flow. The fluid axial velocity increases whereas the fluctuations decreases due to high mass loading in 3-way coupling. The average number of particles tend to increase along the centerline in the 3-way coupled flow. The results gives the insight to further investigate and parameterize the model by varying the stokes number, density, Reynolds number etc.

\section{References}

[1] Longmire, E.K. \& Eaton, J.K., Structure of particle laden jet. J Fluid Mech, 236, pp. 217-258, 1992.

[2] Kulick, J.D., Fessler, J.R. \& Eaton, J.K., Particle response and turbulent modification in fully developed channel flow. J Fluid Mech, 277, pp. 109-134, 1994.

[3] Boivin, M., Simonin, O. \& Squires, K., On the prediction of gas-solid flows with two-way coupling using large eddy simulation. Phys Fluids, 12(8), pp. 2080-2090, 2000.

[4] Jin, H.H., Luo, K., Fan, J.R. \& Cen, K., Large eddy simulation of a particle laden turbulent plane jet. Journal of Zhejiang Uni Sci, 4(2), pp. 175-180, 2003.

[5] Tanaka, T. \& Tsuji, Y., Numerical simulation of gas solid two phase flow in vertical pipe: on the effect of inter-particle collision. ASME/FED gas-solid flows, 121, pp. 123-128, 1991.

[6] Sommerfeld, M. \& Zivkovic, G., "Recent advancements in numerical simulations of pneumatic conveying through pipe system". In: Hirsch, Ch., Periaux, J., Onate, E. (Eds), Comp Meth in Appl Sci, invited lectures and special tech sessions of the First European Comp Fluid Dynamics Conf and the First European Conf on N Methods in Engg, pp. 201-212, 1992.

[7] Oesterle, B. \& Petitjean, A., Simulation of particle to particle interaction in gas solid flows. 19, pp. 199-211, 1993.

[8] Lun, C.K.K. \& Lui, H.S., Numerical simulation of dilute turbulent gas solid flows in horizontal channels. 23, pp. 575-605, 1997.

[9] Sakiz, M., Simonin, O., 2001 "Continuum modelling and Lagrangian simulation of massive frictional colliding particles in a vertical gas-solid channel flow". In: Michaelides, E. (Ed.), Proceedings of the Fourth International Conference on Multiphase Flow, New Orleans, USA (CD-ROM proc. ICMF_2001, Paper 186).

[10] Sommerfeld, M., Analysis of collision effects for turbulent gas particle flow in horizontal channel: Part1. particle transport. 29.

[11] Sommerfeld, M., Validation of stochastic Lagrangian modelling approach for inter-particle collisions in homogeneous isotropic turbulence. 27. 
[12] Yan, J., Luo, K., Tsuji, Y. \& Cen, K., Direct numerical simulation of particle dispersion in a turbulent jet considering inter-particle collisions. Int $J$ Multiphase Flow, 34, pp. 723-733, 208.

[13] Prahl, L., Holzer, A., Arlov, D., Revstedt, J., Sommerfeld, M. \& Fuchs, L., A study of the interaction between two fixed spherical particles. Int $J$ Multiphase Flow, 33, pp. 707-725, 2007.

[14] Prahl, L., Jadoon, A. \& Revstedt, J., Interaction between two spheres placed in tandem arrangement in steady and pulsating flow. Accepted for publication in Int J of Multi-phase flows, May 2009.

[15] Jadoon, A., Prahl, L. \& Revstedt, J., Dynamic interaction of fixed dual spheres for several configurations and inflow conditions. Submitted for publication in Eurpeon $J$ of Mech, 2008.

[16] Salewski, M. \& Fuchs, L., Effects of aerodynamic particle interaction in turbulent non-dilute particle laden flow. $J$ of Turbulence, 9, pp. N-46, 2008.

[17] Rai, M.M. \& Moin, P., Direct simulations of turbulent flow using finitedifference schemes. J Comput Phys, 96, pp. 15-53, 1991.

[18] Schiller \& Nauman, A.Z., A drag coefficient correlation. Ver Deut Ing, 77, pp. 318-320, 1933.

[19] Olsson, P.J. \& Fuchs, L., Large eddy simulation of the proximal region of spatially developing circular jet. Phys Fluids, 8, pp. 2125-2137, 1996. 